\title{
Filigrane
}

Écoutes psychothérapiques

\section{Les émotions au coeur de la santé. Mieux connaître nos émotions pour s'en faire des alliées, de Monique Brillon Monique Brillon, 2009, Les émotions au coeur de la santé. Mieux connaître nos émotions pour s'en faire des alliées, Les éditions de l'Homme, Montréal, 248 p.}

\section{Marie-Ange Pongis-Khandjian}

Volume 19, numéro 1, printemps 2010

Adieu Oedipe, bonjour Narcisse?

URI : https://id.erudit.org/iderudit/044183ar

DOI : https://doi.org/10.7202/044183ar

Aller au sommaire du numéro

Éditeur(s)

Revue Santé mentale au Québec

ISSN

1192-1412 (imprimé)

1911-4656 (numérique)

Découvrir la revue

Citer ce compte rendu

Pongis-Khandjian, M.-A. (2010). Compte rendu de [Les émotions au coeur de la santé. Mieux connaître nos émotions pour s'en faire des alliées, de Monique Brillon / Monique Brillon, 2009, Les émotions au coeur de la santé. Mieux connaître nos émotions pour s'en faire des alliées, Les éditions de l'Homme, Montréal, 248 p.] Filigrane, 19(1), 109-111. https://doi.org/10.7202/044183ar d'utilisation que vous pouvez consulter en ligne. 


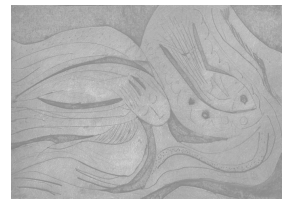

\section{Les émotions au cœur de la santé. Mieux connaître nos émotions pour s'en faire des alliées, de Monique Brillon ${ }^{1}$}

Marie-Ange Pongis-Khandjian

$\mathrm{D}$ octeur en psychologie et psychothérapeute psychanalytique, Monique Brillon a enseigné dans différentes universités du Québec. Elle est aussi l'auteur de: Le labyrinthe de la Féminité (2008), et de La pensée qui soigne. Que savons-nous du pouvoir des émotions (2006), édités tous deux aux Éditions de l'Homme. La fracture de l'oeil (2006), un roman, est édité aux Herbes Rouges.

Dans son dernier livre Les émotions au cour de la santé. Mieux connaître nos émotions pour s'en faire des alliées, Monique Brillon approfondit et peaufine une vision psychosomatique de l'être humain, thème sur lequel elle réfléchit depuis plusieurs années déjà. Cet essai pousse encore plus loin les pistes de réflexion proposées dans son précédent ouvrage, La pensée qui soigne.

Dans ce dernier essai, elle met en évidence, avec un réel talent pédagogique, la relation complexe et intime entre corps et psyché et le trait d'union entre chair et esprit que sont les émotions et les sentiments, faisant de l'être humain un être psychosomatique par essence. Elle se situe au cour d'un mouvement de rassemblement de connaissances plurielles et fait de nombreux liens entre théories et pratique pour améliorer la compréhension et les soins de la personne souffrante.

L'essai est composé de douze chapitres. Chacun d'eux s'ouvre sur une citation provenant d'ouvrages littéraires que l'auteur a aimés et qu'elle nous donne le goût de découvrir - un détail peut-être mais qui donne une idée de l'étendue de sa culture dans des domaines fort divers et qui met en appétit notre pensée de l'imaginaire!

Nous sommes invités à mieux connaître le monde fascinant des émotions. L'auteur insiste sur le fait qu'une émotion n'est à priori ni positive ni négative; elle existe tout simplement et a sa raison d'être au moment où elle se présente. Les émotions nous permettent de nous garder en santé en régulant la tension interne. La régulation affective a la charge d'optimiser les émotions agréables et de minimiser les émotions dérangeantes. 
La différence entre "pensée positive» et «pensée qui soigne» se situe dans le fait que la première valorise les émotions agréables et combat celles qui dérangent principalement par autosuggestion. La pensée de l'imaginaire, elle, ou "pensée qui soigne », outil de l'hémisphère droit du cerveau, rend possible un travail mental, garant de la santé du corps.

L'émotion affecte le corps; grâce au sentiment, trait d'union entre corps et esprit, et aux différentes modalités de la mémoire - implicite, explicite et d'état, mémoire, les deux instruments de la "pensée qui soigne», un travail mental efficace peut se déployer. La fluidité de la circulation entre cerveau et corps est indispensable pour que les images mentales de l'imaginaire puissent être créatives.

Le sommeil est indispensable à une bonne santé physique et psychique, et le rêve, usine de transformations des émotions est l'organisateur psychosomatique par excellence.

L'être humain utilise toutes sortes de stratégies pour contrer les émotions dérangeantes. Il s'agit des mécanismes de défense qui, s’ils sont trop rigides, font que la pensée ne soigne plus et tourne en rond.

Les émotions servent de repères à la pensée qui cherche à comprendre, à interpréter, à donner un sens aux changements constants qui nous entourent; c'est un processus essentiel à l'adaptation et à la construction de l'identité. Les sens naissent, évoluent, disparaissent et se succèdent; s'y cramponner, ou ne pas en trouver, bloque le processus de pensée et cause de la souffrance aussi bien physique que morale, inhérente à la nature humaine, impossible à éviter et à éliminer. Elle naît dans l'intersubjectif mais c'est aussi dans une relation qu'elle peut se résoudre.

Les expressions les plus répandues du mal-être psychosomatique sont la dépression, le burn-out et la fatigue chronique. Le rôle que joue la mise à l'écart des émotions dans l'apparition de ces déséquilibres est examiné, ainsi que la façon de retrouver le bien-être par l'harmonisation d'une approche médicamenteuse et psychothérapeutique.

La douleur, à la fois réalité biologique et construction subjective de l'esprit, entretient avec la mémoire et les émotions des liens étroits. L'effet placebo et les facteurs psychologiques et relationnels sont susceptibles de l'influencer dans certaines guérisons inexplicables par la médecine. L'auteur passe en revue les principales composantes physiologiques et psychiques des processus de guérison et clarifie aussi les limites de la pensée soignante. Elle interroge aussi, dans un esprit d'ouverture et de curiosité critique, des domaines tels la méditation, l'hypnose, la perception extrasensorielle, les rêves prémonitoires, mais aussi la physique quantique et la théorie de l'information.

L'auteur propose enfin des moyens pour exercer sa pensée soignante: méditation, hypnose, méthode en écho, EMDR, imagerie mentale, psychothérapie psychanalytique psychosomatique et psychanalyse.

Tout au long de cet ouvrage, s'appuyant sur deux champs théoriques - les neurosciences et la psychosomatique d'inspiration psychanalytique - et les entre-tissant de façon subtile, Monique Brillon illustre de façon claire comment les neurosciences 
fournissent un fond biologique et un appui aux théories psychanalytiques et permettent de les revisiter pour arriver à une meilleure compréhension du fonctionnement psychique humain. Elle montre comment la biologie confirme l'importance des facteurs psychologiques dans le maintien de la santé et nous met en garde paradoxalement contre une tendance à trop biologiser la façon de soigner les souffrances de l'âme.

Nous sommes loin de toute psycho-pop aussi bien que des méthodes de guérison miraculeuses. Bien au contraire, l'auteure nous convainc que les émotions dites «négatives» sont indispensables à notre survie et à un bon fonctionnement psychique pour autant que nous arrivions à composer avec elles et qu'elles puissent être canalisées par le travail mental de la pensée imaginaire qui soigne. Elle propose différentes voies à explorer pour tenter d'y arriver, à chacun de trouver celle qui lui convient le mieux.

Ce livre s'adresse à tout intervenant en santé, tant physique que mentale, ainsi qu'à un public plus large. Le style est vivant et des questions directes au lecteur l'obligent à se questionner de façon personnelle. Chacun y trouvera matière à réflexion et à approfondissement. Des exemples cliniques et des schémas illustrent de façon claire le travail de l'imaginaire, celui de la pensée qui soigne.

Marie-Ange Pongis-Khandjian psychologue et psychanalyste

\section{Note}

1. Monique Brillon, 2009, Les émotions au cour de la santé. Mieux connaître nos émotions pour s'en faire des alliées, Les éditions de l'Homme, Montréal, 248 p. 